\title{
Pseudothrombus - An Echocardiographic Illusion
}
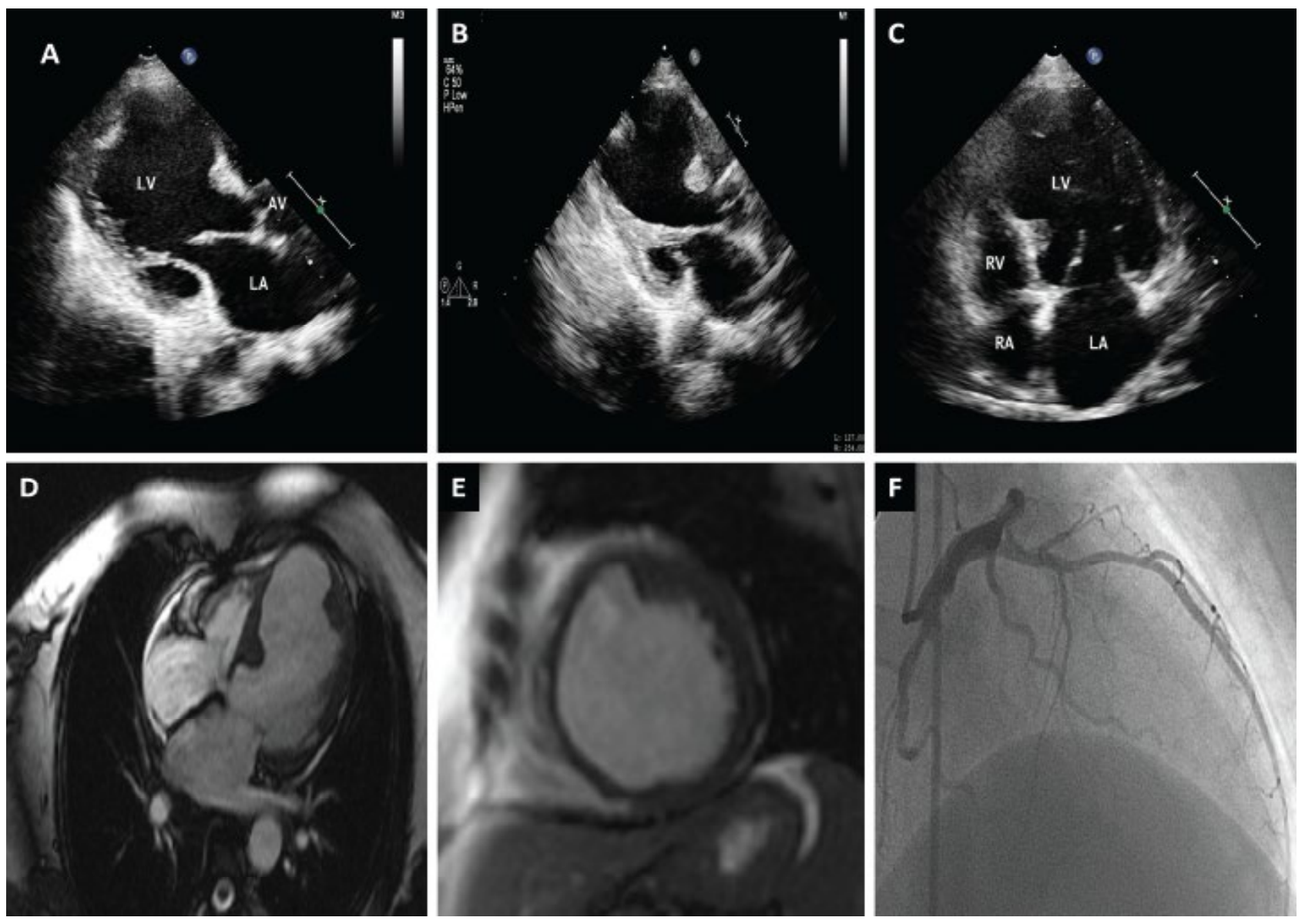

Cardiovascular complications like hypertension, premature atherosclerosis, coronary arteritis and myocarditis are not infrequent in systemic lupus erythematosus (SLE). There are case reports describing SLE associated left ventricular (LV) aneurysms secondary to myocarditis or more commonly post left ventricular infarction. We report one such case of a 45 years old lady in quiescent phase of SLE who presented with exertional dyspnea for six months. Clinical examination was unremarkable with normal blood pressure. Two dimensional echocardiography (2D Echo) revealed LV aneurysm involving mid and apical anterior and anteroseptal segments with two masses seen along the margin of this aneurysmal segment (Panel A, Panel B and Panel C). Due to unusual location of these masses along the aneurysmal margin and their homogeneous echogenecity, cardiac magnetic resonance imaging (CMR) was planned which revealed these masses to be localised myocardial thickenings secondary to remodelling (Panel D, Panel E and Supplementary video). Coronary angiogram was suggestive of insignificant stenosis of left anterior descending (LAD) artery and patient was advised guideline based medical therapy (Panel F).

Post infarction LV remodelling commonly results in left ventricular dilatation and hypertrophy however localised myocardial thickening along aneurysmal segment margin is uncommon. Moreover such thickening simulates thrombus on echocardiography leading to unindicated anticoagulation.

\section{Information}

\section{Rishi Vinod Lohiya* and Sunil Washimkar}

Department of Cardiology, Post Graduate Institute \& Super speciality Hospital, India

*Correspondence: Rishi Vinod Lohiya, Department of Cardiology, Post Graduate Institute \& Superspeciality Hospital, Nagpur, Maharashtra, India, Tel: 919320182115, Email: rishidr123@rediffmail.com
Citation: Lohiya RV, Washimkar S (2016) Pseudothrombus An Echocardiographic Illusion. Clin Med Img Lib 2:043

Published: May 16, 2016

Copyright: () 2016 Lohiya RV, et al. This is an open-access content distributed under the terms of the Creative Commons Attribution License, which permits unrestricted use, distribution, and reproduction in any medium, provided the original author and source are credited.

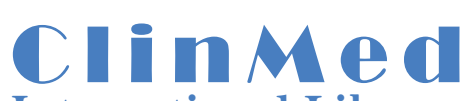

International Library 